\title{
Enhanced spin Hall effect by tuning antidot potential: Proposal for a spin filter
}

\author{
Tomohiro Yokoyama* and Mikio Eto \\ Faculty of Science and Technology, Keio University, 3-14-1 Hiyoshi, Kohoku-ku, Yokohama 223-8522, Japan
}

(Dated: November 10, 2018)

\begin{abstract}
We propose an efficient spin filter including an antidot fabricated on semiconductor heterostructures with strong spin-orbit interaction. The antidot creates a tunable potential on two-dimensional electron gas in the heterostructures, which may be attractive as well as repulsive. Our idea is based on the enhancement of extrinsic spin Hall effect by resonant scattering when the attractive potential is properly tuned. Numerical studies for three- and four-terminal devices indicate that the efficiency of the spin filter can be more than $50 \%$ by tuning the potential to the resonant condition.
\end{abstract}

PACS numbers: 72.25.Dc,71.70.Ej,73.23.-b,85.75.-d

\section{INTRODUCTION}

The injection and manipulation of electron spins in semiconductors are important issues for spin-based electronics, "spintronics." portant role in the manipulation of the spins. For conduction electrons in direct-gap semiconductors, the SO interaction is expressed in the same form as that in vacuum, that is,

$$
H_{\mathrm{SO}}=\frac{\lambda}{\hbar} \sigma \cdot[\boldsymbol{p} \times \boldsymbol{\nabla V}(\boldsymbol{r})]
$$

where $V(\boldsymbol{r})$ is an external potential and $\sigma$ indicates the electron spin $s=\sigma / 2$. The coupling constant $\lambda$ is significantly enhanced by the band effect, particularly in narrow-gap semiconductors such as InAs, 2 compared with that in vacuum, $|\lambda|=\hbar^{2} /\left(4 m_{0}^{2} c^{2}\right)$ with $m_{0}$ as the electron mass and $c$ as the velocity of light.

In two-dimensional electron gas (2DEG) in semiconductor heterostructures, an electric field perpendicular to the $2 \mathrm{DEG}$ results in the Rashba SO interaction $\stackrel{\underline{3}}{=}$ For the electric field $\mathcal{E}$ in the $z$ direction, the substitution of $V(\boldsymbol{r})=e \mathcal{E} z$ into Eq. (1) yields

$$
H_{\text {SO }}=\frac{\alpha}{\hbar}\left(p_{y} \sigma_{x}-p_{x} \sigma_{y}\right),
$$

with $\alpha=e \mathcal{E} \lambda$. Large values of $\alpha$ have been reported in experiments. ${ }^{4.5 .6}$ In the spin transistor proposed by Datta and Das, $\underline{\underline{7}}$ electron spins are injected into the semiconductor heterostructures from a ferromagnet, and manipulated by tuning the strength of Rashba SO interaction by adjusting the electric field $\mathcal{E}$. The spins are detected by another ferromagnet. It is well-known, however, that the efficiency of spin injection from a ferromagnetic metal to semiconductors is very poor, less than $0.1 \%$, due to the conductivity mismatch $\stackrel{8}{=}$ To overcome this difficulty, the SO interaction may be useful for the efficient spin injection, besides the spin manipulation, in the spin transistor. Several spin filters have been proposed utilizing the SO interaction, e.g., three- or four-terminal devices based on the spin Hall effect (SHE), 9,10,11,12 a triple-barrier tunnel diode,,$\underline{13}$ a quantum point contact, ${ }^{14,15}$ a three-terminal device for the Stern-Gerlach experiment using a nonuniform SO interaction,,$\underline{16}$ and an open quantum dot,$\frac{17}{}$ Yamamoto and Kramer proposed a three-terminal spin filter with an antidot, using a SHE caused by the scattering of electrons at a repulsive potential created by the antidot. $\frac{18}{}$

The SHE is one of the phenomena to create a spin current due to the SO interaction. There are two types of SHE. One is an intrinsic SHE which creates a dissipationless spin current in the perfect crystal $\stackrel{19,20}{ }$ Murakami et al., for example, proposed that the drift motion of holes in SO-split valence bands induces an intrinsic SHE $\stackrel{19}{\underline{19}}$ The SHE of the hole system has been observed experimentally by Wunderlich et al., using a $p$ - $n$ junction light-emitting diode 21 The other type is an extrinsic SHE caused by the spin-dependent scattering of electrons by impurities $\stackrel{22,23,24,25}{ }$ For a centrally symmetric potential around an impurity, $V(r)\left(r=\sqrt{x^{2}+y^{2}+z^{2}}\right)$, Eq. (1) is rewritten as

$$
H_{\mathrm{SO}}=-\lambda \frac{2}{r} \frac{d V}{d r} \boldsymbol{l} \cdot \boldsymbol{s}
$$

where $\boldsymbol{l}=(\boldsymbol{r} \times \boldsymbol{p}) / \hbar$ is the angular momentum. This results in the skew scattering: accompanied by the scattering from direction $\boldsymbol{n}$ to $\boldsymbol{n}^{\prime}$, the spin is polarized in $\left(\boldsymbol{n} \times \boldsymbol{n}^{\prime}\right) /\left|\boldsymbol{n} \times \boldsymbol{n}^{\prime}\right|{ }^{26,27}$ In an optical experiment on the Kerr rotation, Kato et al. observed a spin accumulation at sample edges along the electric current in $n$-type GaAs, $\stackrel{28}{n}$ which is ascribable to the extrinsic SHE. The experimental result has been quantitatively explained by a semi-classical theory considering the skew scattering and "side jump" effects. ${ }^{25}$

In our previous paper, ${ }^{29}$ we have quantum-mechanically formulated the extrinsic SHE for 2DEG in semiconductor heterostructures. We have examined the SHE due to the scattering by an artificial potential created by an antidot, scanning tunnel microscope (STM) tip, etc. An antidot is a small metallic electrode fabricated above 2DEG, which creates an electrically tunable potential on 2DEG. The potential is usually repulsive, but it could be attractive when a positive voltage is applied to the antidot. We have found that the SHE is significantly enhanced by resonant scattering when the attractive potential is properly tuned.

We have stressed that the extrinsic SHE is easier to understand in 2DEG than in three-dimensional case. Let us consider an axially symmetric potential $V(r)\left(r=\sqrt{x^{2}+y^{2}}\right)$ created by an antidot on conduction electrons in the $x y$ plane. The SO interaction in Eq. (1) becomes

$$
H_{\mathrm{SO}}=-\lambda \frac{2}{r} \frac{d V}{d r} l_{z} s_{z} \equiv V_{1}(r) l_{z} s_{z},
$$


(A)
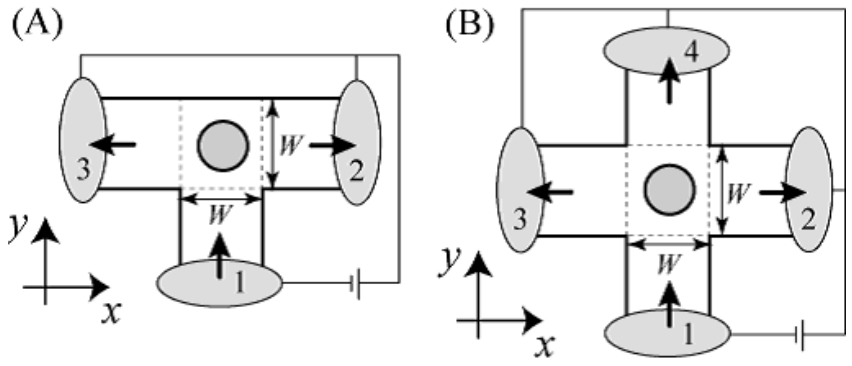

FIG. 1: Our model for (A) three- and (B) four-terminal devices for the spin filter. They are fabricated on two-dimensional electron gas in the $x y$ plane. Both the devices include an antidot at the center of junction, which is a square area surrounded by broken lines. Three or four ideal leads connect the junction to reservoirs. Reservoir 1 is a source from which unpolarized electrons are injected into the junction. The voltages are equal in the drains; reservoirs 2 and 3 in model (A) and reservoirs 2, 3, and 4 in model (B).

where $l_{z}$ and $s_{z}$ are the $z$ component of angular momentum and spin operators, respectively. $V_{1}(r)=-(2 \lambda / r) V^{\prime}(r)$, which has the same sign as $V(r)$ if $|V(r)|$ is a monotonically decreasing function of $r$ and $\lambda>0$. Assuming that $V(r)$ is smooth on the scale of the lattice constant, we adopt the effective mass equation

$$
\left[-\frac{\hbar^{2}}{2 m^{*}} \Delta+V(r)+V_{1}(r) l_{z} s_{z}\right] \psi(\boldsymbol{r})=E \psi(\boldsymbol{r}),
$$

for an envelope function $\psi(\boldsymbol{r})$ with $m^{*}$ as the effective mass. In Eq. (5), $l_{z}$ and $s_{z}$ are conserved in contrast to the threedimensional case with Eq. (3). For $s_{z}= \pm 1 / 2$, an electron experiences the potential of $V(r) \pm V_{1}(r) l_{z} / 2$. As a consequence, the scattering of components of $l_{z}>0\left(l_{z}<0\right)$ is enhanced (suppressed) by the SO interaction for $s_{z}=1 / 2$ when $V_{1}(r)$ has the same sign as $V(r)$. The effect is opposite to that for $s_{z}=-1 / 2$. This is the origin of the extrinsic SHE. We have formulated the SHE in terms of phase shifts in the partial wave expansion for 2DEG and shown that the SHE is largely enhanced by resonant scattering .29 These results are summarized in Appendix A.

In the present paper, we consider three- and four-terminal devices including an antidot, as shown in Fig. 11 and examine the enhancement of the SHE. We evaluate an efficiency of the spin-filtering effect by resonant scattering in the case of attractive potential. Although our three-terminal device is very similar to the spin filter proposed by Yamamoto and Kramer, $\frac{18}{18}$ they have only studied the case of repulsive potential. We show that our device can be a spin filter with an efficiency of more than $50 \%$ by tuning the potential to the resonant condition.

We numerically solve the effective mass equation [Eq. (5)] with an appropriate boundary condition for our devices. A confining potential for the leads (quantum wires) could induce the SO interaction, following Eq. (1) ? $^{30,31,32,33}$ However, its effect on the electrons is much smaller than the SO interaction induced by the antidot potential because the amplitude of the wavefunction is small around the edges of the leads. There- fore, we consider the antidot-induced SO interaction only. We also assume that the antidot potential $V(\boldsymbol{r})$ is independent of $z$. Otherwise, it would create the Rashba-type SO interaction, Eq. (2) with $\alpha=\lambda(\partial V / \partial z)$, in addition to Eq. (4). The Dresselhaus SO interaction is also disregarded, which is induced by the inversion asymmetry of the crystal ${ }^{34}$ These effects will be discussed in the final section.

The electron-electron interaction is not taken into account. The Coulomb blockade is not relevant to the case of antidot, in contrast to that of conventional quantum dot which is connected to the leads via tunnel barriers $\frac{35}{\sqrt{5}}$ In our model, therefore, the influence of the electron-electron interaction is only quantitative and could be considered by the mean-field level, as discussed in the final section. Note that there have been several researches for the spin-current generation based on the electron-electron interaction in the absence of SO interaction, e.g., using single or double quantum dots ${ }^{36,37,38}$ and quantum wires $39,40,41,42,43$

The organization of the present paper is as follows. In Sec. II, we describe our model for three- and four-terminal devices and calculation method. The calculation of spin-dependent conductance in multi-terminal devices is formulated using the Green's function in the tight-binding model. ${ }^{44,45,46,47}$ In Sec. III, we present numerical results of the conductance and spinfiltering effect when the strength of antidot potential is tuned. We also investigate the density of states (DOS) in the junction area of the devices to illustrate virtual bound states. The existence of the virtual bound states at the Fermi level is a strong evidence that a resonant scattering takes place when the spinfiltering effect is remarkably enhanced. In addition, we perform a channel analysis of the spin-dependent conductance to closely examine the resonance. The final section (Sec. IV) is devoted to the conclusions and discussion.

\section{MODEL AND CALCULATION METHOD}

In this section, we explain our model and calculation method. We numerically solve the effective mass equation in the tight-binding model for the devices. In the presence of the SO interaction in Eq. (4), the $z$ component of electron spin $s_{z}$ is conserved although $l_{z}$ is not a good quantum number owing to the lack of rotational symmetry in our devices. Hence we can solve the equation for $s_{z}= \pm 1 / 2$ separately.

\section{A. Model}

We consider three- and four-terminal devices with an antidot, fabricated on semiconductor heterostructures, as shown in Fig. 1. Three or four leads (quantum wires) of width $W$ are joined to one another at a junction, which is a square area surrounded by broken lines in the figure. The leads are formed by hard-wall potential and connected to the reservoirs. Reservoir 1 is a source from which unpolarized electrons are injected into the junction. The electrons are outgoing to the drains; reservoirs 2 and $3(2,3$, and 4$)$ in the three-terminal (fourterminal) device. The voltages are equal in all the drains. 
An antidot creates an axially symmetric potential $V(r)$, where $r$ is the distance from the center of the junction. It is assumed to be attractive and given by a smooth potential well,

$$
V(r)= \begin{cases}V_{0} & \left(r-R_{0}<-\frac{\Delta R_{0}}{2}\right) \\ \frac{V_{0}}{2}\left\{1-\sin \left(\pi \frac{r-R_{0}}{\Delta R_{0}}\right)\right\} & \left(\left|r-R_{0}\right| \leq \frac{\Delta R_{0}}{2}\right) \\ 0 & \left(r-R_{0}>\frac{\Delta R_{0}}{2}\right)\end{cases}
$$

with $V_{0}<0$. The radius of the potential well is $R_{0}=W / 4$, and we choose $\Delta R_{0}=0.7 R_{0}$. The gradient of $V(r)$ gives rise to the $\mathrm{SO}$ interaction in Eq. (4).

For the numerical study, we discretize the two-dimensional space with lattice constant $a$ (tight-binding model with a square lattice). The width of the leads is $W=(N+1) a$ with $N=29$; the wavefunction becomes zero at the zeroth and $(N+1)$ th sites in the transverse direction of the leads. The Hamiltonian is

$$
\begin{aligned}
H & =t \sum_{i, j, \sigma}\left(4+\tilde{V}_{i, j}\right) c_{i, j ; \sigma}^{\dagger} c_{i, j ; \sigma} \\
& -t \sum_{i, j, \sigma}\left(T_{i, j ; i+1, j ; \sigma} c_{i, j ; \sigma}^{\dagger} c_{i+1, j ; \sigma}\right. \\
& \left.+T_{i, j ; i, j+1 ; \sigma} c_{i, j, \sigma}^{\dagger} c_{i, j+1, \sigma}+\text { h. c. }\right)
\end{aligned}
$$

where $c_{i, j ; \sigma}^{\dagger}$ and $c_{i, j ; \sigma}$ are creation and annihilation operators of an electron at site $(i, j)$ with spin $\sigma$. Here, $t=\hbar^{2} / 2 m^{*} a^{2}$, where $m^{*}$ is the effective mass of electrons. $\tilde{V}_{i, j}$ is the potential energy at site $(i, j)$, in units of $t$. The transfer term in the $x$ direction is given by

$$
T_{i, j ; i+1, j ; \pm}=1 \pm i \tilde{\lambda}\left(\tilde{V}_{i+1 / 2, j+1}-\tilde{V}_{i+1 / 2, j-1}\right)
$$

whereas that in the $y$ direction is

$$
T_{i, j ; i, j+1 ; \pm}=1 \mp i \tilde{\lambda}\left(\tilde{V}_{i+1, j+1 / 2}-\tilde{V}_{i-1, j+1 / 2}\right),
$$

where $\tilde{\lambda}=\lambda /\left(4 a^{2}\right)$ is the dimensionless coupling constant of the SO interaction. $\tilde{V}_{i+1 / 2, j}$ is the average of the potential energy at sites $(i, j)$ and $(i+1, j)$, and $\tilde{V}_{i, j+1 / 2}$ is that at sites $(i, j)$ and $(i, j+1)$.

The SO interaction is absent in the leads. The wavefunction of conduction channel $\mu$ in the leads is written as

$$
\begin{aligned}
\psi_{\mu}\left(i^{\prime}, j^{\prime}\right) & =\exp \left(i k_{\mu} a j^{\prime}\right) u_{\mu}\left(i^{\prime}\right), \\
u_{\mu}\left(i^{\prime}\right) & =\sqrt{\frac{2}{N+1}} \sin \left(\frac{\pi \mu i^{\prime}}{N+1}\right),
\end{aligned}
$$

where $i^{\prime}$ and $j^{\prime}$ denote the site numbers in the transverse and longitudinal directions of the leads, respectively. The wavenumber $k_{\mu}$ is determined from the condition that the dispersion relation

$$
E_{\mu}(k)=4 t-2 t \cos \left(\frac{\pi \mu}{N+1}\right)-2 t \cos (k a)
$$

is identical to the Fermi energy $E_{\mathrm{F}}$. The band edge of channel $\mu$ is defined by $E_{\mu}(k=0)$. The band edge is located below $E_{\mathrm{F}}$ for the conduction channel. For $E_{\mu}(k=0)>E_{\mathrm{F}}$, on the other hand, mode $\mu$ is an evanescent mode. The wavefunction of the evanescent mode is given by

$$
\psi_{\mu}\left(i^{\prime}, j^{\prime}\right)=\exp \left(-\kappa_{\mu} a j^{\prime}\right) u_{\mu}\left(i^{\prime}\right),
$$

where $a j^{\prime}$ is the distance from the junction along the lead and $\kappa_{\mu}$ satisfies $E_{\mu}\left(i \kappa_{\mu}\right)=E_{\mathrm{F}}$.

\section{B. Calculation method}

For numerical studies, we introduce the Green's function. The Green's function for the junction area is defined by

$$
\hat{G}_{\sigma}(E)=\left[E I-\mathcal{H}_{\sigma}-\sum_{p} \Sigma^{p}\right]^{-1},
$$

where $\mathcal{H}_{\sigma}$ is the truncated matrix of the Hamiltonian for the junction area $\left(N^{2} \times N^{2}\right)$ with spin $\sigma$, and $\Sigma^{p}$ is the self-energy due to the coupling to the lead $p$ :

$$
\Sigma^{p}=-t \tau_{p}^{\dagger} U \Lambda U^{-1} \tau_{p} .
$$

Here $U$ is a unitary matrix, $U=\left(\boldsymbol{u}_{1}, \boldsymbol{u}_{2}, \cdots, \boldsymbol{u}_{N}\right)$, where $\boldsymbol{u}_{\mu}=$ $\left[u_{\mu}(1), u_{\mu}(2), \cdots, u_{\mu}(N)\right]^{t} \cdot \Lambda=\operatorname{diag}\left(\lambda_{1}, \lambda_{2}, \cdots, \lambda_{N}\right)$, where $\lambda_{\mu}=\exp \left(i k_{\mu} a\right)$ for conduction channels and $\lambda_{\mu}=\exp \left(-\kappa_{\mu} a\right)$ for evanescent modes. $\tau_{p}$ is a coupling matrix $\left(N \times N^{2}\right)$ between lead $p$ and the junction: $\tau_{p}\left(p_{i}, i\right)=1$ for $p_{i}$ being an adjacent site in lead $p$ to site $i$ in the junction; $\tau_{p}\left(p_{i}, i\right)=0$ otherwise..$^{44}$

The spin-dependent conductance from reservoir $p$ to reservoir $q$ is obtained from the Landauer-Büttiker formula. It is written as

$$
G_{\sigma}^{q p}=\frac{e^{2}}{h} \operatorname{Tr}\left[\Gamma^{q} \hat{G}_{\sigma}(E) \Gamma^{p} \hat{G}_{\sigma}^{\dagger}(E)\right],
$$

where

$$
\Gamma^{p}=i\left[\Sigma^{p}-\Sigma^{p^{\dagger}}\right]
$$

The total conductance is $G^{q p}=G_{+}^{q p}+G_{-}^{q p}$, whereas the spin polarization in the $z$ direction is defined by

$$
P_{z}^{q p}=\frac{G_{+}^{q p}-G_{-}^{q p}}{G_{+}^{q p}+G_{-}^{q p}}
$$

for the current from reservoir $p$ to $q$.

To elucidate the virtual bound states in the potential well, we calculate the DOS in the junction area. It is evaluated from the Green's function (14) as ${ }^{48}$

$$
D(E)=-\frac{1}{\pi} \sum_{\sigma} \operatorname{Im}\left[\operatorname{Tr} \hat{G}_{\sigma}(E)\right] .
$$

We assume that $\tilde{\lambda}=0.1$ for the strength of SO interaction, which corresponds to the value for InAs, $\lambda=117.1 \AA^{2}, \frac{2}{2}$ with $a=W / 30$ and width of the leads $W \approx 50 \mathrm{~nm}$. The temperature $T=0$. We focus on the transport from reservoir 1 to 2 and omit the superscript 21 of $G_{ \pm}^{21}$ and $P_{z}^{21}$, otherwise stated. Note that the conductance from reservoir 1 to 3 is related to $G_{ \pm}^{31}=$ $G_{\mp}^{21}$ from the symmetry of the system, for both three- and fourterminal devices. The current from reservoir 1 to 4 is not spinpolarized in the four-terminal device. 

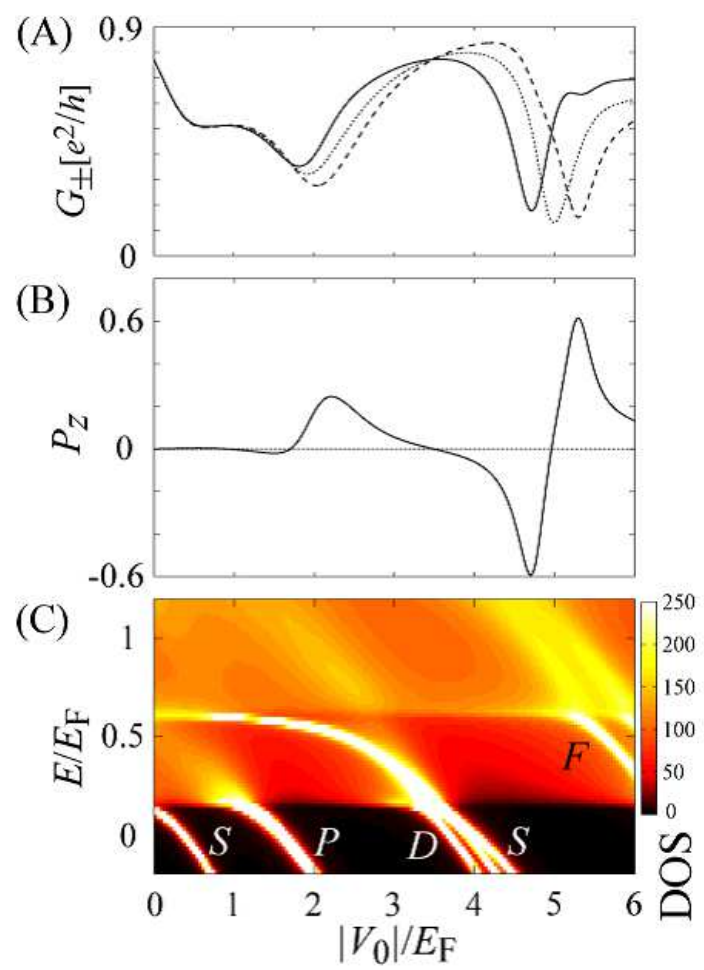

FIG. 2: (Color online) Numerical results for the three-terminal device with $k_{\mathrm{F}} R_{0}=2$, where $R_{0}$ is the radius of attractive potential. (A) Conductance $G_{ \pm}$from reservoir 1 to 2 in Fig. 11 A) for $s_{z}= \pm 1 / 2$ and (B) spin polarization $P_{z}$ of the output current in reservoir 2, as functions of the potential depth $\left|V_{0}\right|$. In (A), solid and broken lines indicate $G_{+}$and $G_{-}$, respectively. A dotted line shows the conductance per spin in the absence of the SO interaction. (C) Grayscale plot of the density of states in the junction area, $D(E)$, in the plane of $\left|V_{0}\right|$ and energy $E$ of electron.

\section{CALCULATED RESULTS}

We calculate the conductance $G_{ \pm}$for spin $s_{z}= \pm 1 / 2$ and spin polarization $P_{z}$ when the potential depth $\left|V_{0}\right|$ is tuned. We examine three cases of $k_{\mathrm{F}} R_{0}=1,2$, and 3 , where the Fermi wavenumber $k_{\mathrm{F}}$ is defined by the Fermi energy $E_{\mathrm{F}}$ as $E_{\mathrm{F}} / t=\left(k_{\mathrm{F}} a\right)^{2} \cdot{ }^{49}$ In the three cases, the Fermi energy $E_{\mathrm{F}}$ is different, whereas the radius of the potential well $R_{0}$ is fixed. The number of conduction channels in the leads is 1,2 , and 3 , respectively.

Here we discuss the cases of $k_{\mathrm{F}} R_{0}=2$ and 3. The numerical result with $k_{\mathrm{F}} R_{0}=1$ is given in Appendix B. (Surprisingly, we find a perfect spin polarization $P_{z}=1$ in the case of $k_{\mathrm{F}} R_{0}=1$. However, the transport properties seem quite specific. This is due to a strong interference effect in the case of single conduction channel.)

\section{A. Case of $k_{\mathrm{F}} R_{0}=2$}

We begin with the three-terminal device in the presence of two conduction channels in the leads $\left(k_{\mathrm{F}} R_{0}=2\right)$. Figures 2 (A) and 2(B) show the conductance $G_{ \pm}$for $s_{z}= \pm 1 / 2$ and spin polarization $P_{z}$, respectively, when the potential depth $\left|V_{0}\right|$ is gradually changed. As seen in Fig. 2(A), the conductance $G_{ \pm}$ shows three minima as a function of $\left|V_{0}\right|$. At the first minimum at $\left|V_{0}\right| / E_{\mathrm{F}} \approx 0.6$, the difference in the conductance for $s_{z}=$ $\pm 1 / 2$ is small. At the second and third minima at $\left|V_{0}\right| / E_{\mathrm{F}} \approx 2$ and 5, respectively, the difference is remarkable, which results in a large spin polarization in the $z$ direction [Fig. 2[B)]. $P_{z}$ is enhanced to $25 \%$ around the second minimum and $61 \%$ around the third minimum.

The behavior of $G_{ \pm}$should be ascribable to resonant scattering at the potential well. The resonant scattering takes place through a virtual bound state in the potential well, which enhances the electron scattering to the unitary limit (Appendix A). This makes the minima of $G_{ \pm}$in our situation of both three- and four-terminal devices. (It is not trivial whether the resonant scattering makes minimum or maximum of the conductance in multi-terminal devices. See the discussion in Appendix A.) Around the minima of the conductance, the difference between $G_{+}$and $G_{-}$is magnified. Look at the third minimum of the conductance around $\left|V_{0}\right| / E_{\mathrm{F}} \approx 5$. The minimum of $G_{+}$is located at a smaller value of $\left|V_{0}\right|$ than that of $G_{-}$. In consequence, $P_{z}$ shows a pair of negative $\operatorname{dip}\left(G_{+}<G_{-}\right)$and a positive peak $\left(G_{+}>G_{-}\right)$. This dip-peak structure of $P_{z}$ can be understood in terms of phase shifts when the resonance for $s_{z}= \pm 1 / 2$ is well separated from each other (Appendix A).

To confirm the above-mentioned scenario regarding the resonant scattering, we calculate the DOS in the junction area. Figure 2(C) shows a grayscale plot of the DOS $D(E)$ in the plane of $\left|V_{0}\right|$ and energy $E$ of electron. The band edge for the lowest and second conduction channels in the leads $\left[E_{1}(k=0)\right.$ and $E_{2}(k=0)$ in Eq. [12)] are located at $E / E_{\mathrm{F}}=0.154$ and 0.615 , respectively. The sharp peaks of $D(E)$ below the lowest band edge correspond to the bound states inside the junction area. With an increase in the potential depth $\left|V_{0}\right|$, several bound states appear one after another. The first one is an $S$ like bound state $\left(l_{z}=0\right)$ although the angular momentum $l_{z}$ is not a good quantum number in our device because of the lack of rotational symmetry. The bound state exists even without the potential well $\left(\left|V_{0}\right|=0\right)$ in the junction area, ${ }^{10}$ and changes to the $S$-like bound state in the potential well with increasing $\left|V_{0}\right|$. The state is doubly degenerate due to the Kramers degeneracy. The next are $P$-like bound states $\left(l_{z}= \pm 1\right)$. They are a pair of Kramers degenerate states. Then $D$-like bound states $\left(l_{z}= \pm 2\right)$ appear, which are clearly split into two by the SO interaction. Another $S$-like state is located at approximately the same energy. Finally $F$-like bound states $\left(l_{z}= \pm 3\right)$ appear in Fig. 2 (C). The pair of Kramers degenerate states is largely separated for the $F$-like states.

The peaks of the bound states in $D(E)$ are broadened above the band edge of the lowest conduction channel in the leads, which significantly influence the electron scattering at the Fermi level as virtual bound states. The second and third minima of the conductance $G_{ \pm}$are located at the position of $D$ 

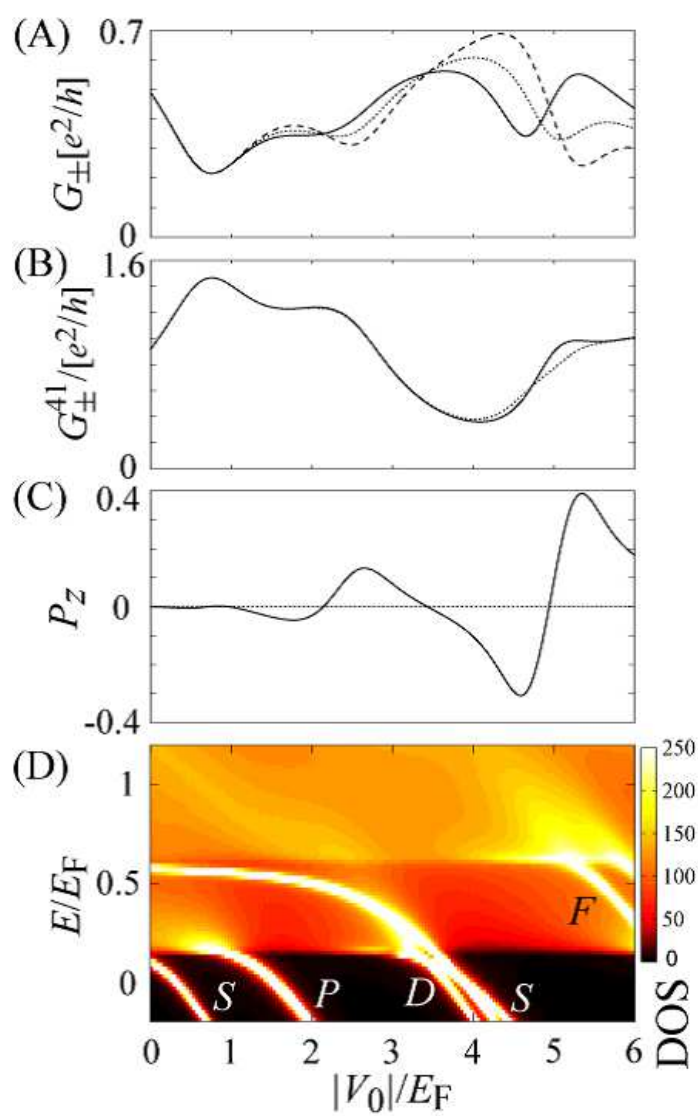

FIG. 3: (Color online) Numerical results for the four-terminal device with $k_{\mathrm{F}} R_{0}=2$, where $R_{0}$ is the radius of attractive potential. (A) Conductance $G_{ \pm}$from reservoir 1 to 2 in Fig.11B) for $s_{z}= \pm 1 / 2$, (B) conductance $G_{ \pm}^{41}$ from reservoir 1 to 4 , and (C) spin polarization $P_{z}$ of the output current in reservoir 2 , as functions of the potential depth $\left|V_{0}\right|$. In (A) and (B), solid and broken lines indicate $G_{+}$and $G_{-}$, or $G_{+}^{41}$ and $G_{-}^{41}$, respectively. A dotted line shows the conductance per spin in the absence of the SO interaction. In (B), solid and broken lines for $G_{ \pm}^{41}$ are completely overlapped. (D) Grayscale plot of the density of states in the junction area, $D(E)$, in the plane of $\left|V_{0}\right|$ and energy $E$ of electron.

and $F$-like virtual bound states at $E_{\mathrm{F}}$, respectively. This is a clear evidence of the resonant scattering through virtual bound states. (At the first minimum of $G_{ \pm}$around $\left|V_{0}\right| / E_{\mathrm{F}}=0.6$, we cannot find any virtual bound state at the Fermi level. The minimum of $G_{ \pm}$may not be due to the resonant scattering, but due to some interference effect around the junction.)

We present the calculated results for the four-terminal device with $k_{\mathrm{F}} R_{0}=2$ in Fig. 3. (A) conductance $G_{ \pm}$for $s_{z}= \pm 1 / 2$ from reservoir 1 to 2 in Fig. 11(B), (B) conductance $G_{ \pm}^{41}$ from reservoir 1 to 4 , and (C) spin polarization $P_{z}$ of the output current in reservoir 2 , as functions of the potential depth $\left|V_{0}\right|$. As seen in Fig. 3 B,$G_{+}^{41}=G_{-}^{41}$ because the SHE does not make a spin polarization in the output current in reservoir 4 . The characters of conductance $G_{ \pm}$for $s_{z}= \pm 1 / 2$ and spin polarization $P_{z}$ are almost the same as those in Fig. 2 for three-terminal device. The conductance shows three min-

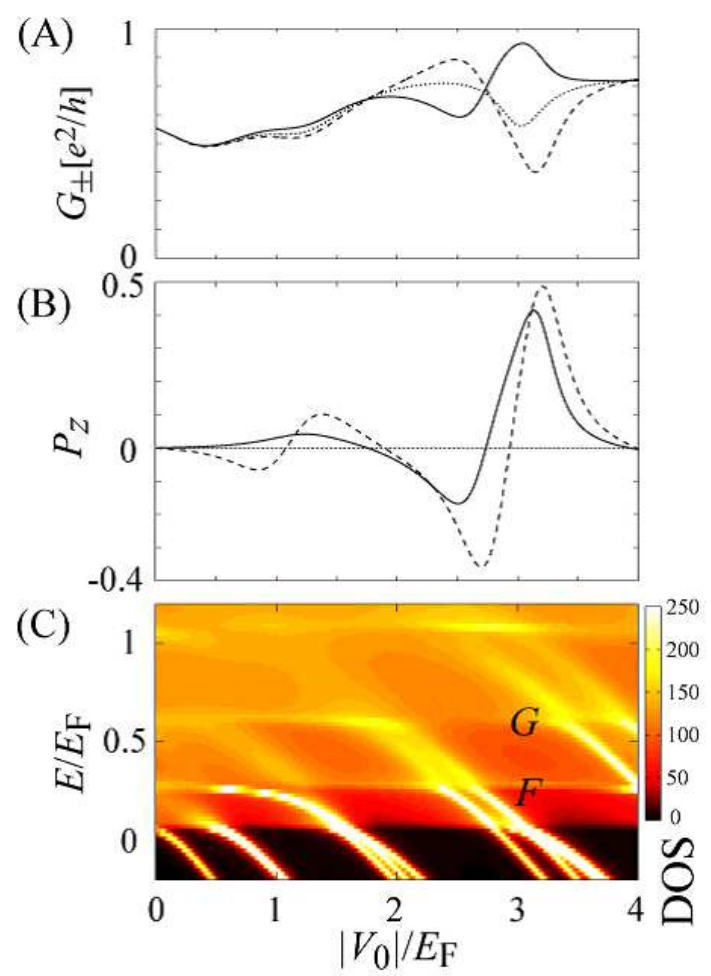

FIG. 4: (Color online) Numerical results for the three-terminal device with $k_{\mathrm{F}} R_{0}=3$, where $R_{0}$ is the radius of attractive potential. (A) Conductance $G_{ \pm}$from reservoir 1 to 2 in Fig. 11 A) for $s_{z}= \pm 1 / 2$ and (B) spin polarization $P_{z}$ of the output current in reservoir 2, as functions of the potential depth $\left|V_{0}\right|$. In (A), solid and broken lines indicate $G_{+}$and $G_{-}$, respectively. A dotted line shows the conductance per spin in the absence of the SO interaction. (C) Grayscale plot of the density of states in the junction area, $D(E)$, in the plane of $\left|V_{0}\right|$ and energy $E$ of electron. Regarding the result for four-terminal device with $k_{\mathrm{F}} R_{0}=3$, a broken line in (B) indicates the spin polarization $P_{z}$ of the output current in reservoir 2 in Fig. 1(B).

ima. The second and third minima are clearly due to resonant scattering via $D$ - or $F$-like virtual bound states, as seen in the DOS in Fig. 3(D). Around the minima, the conductance for $s_{z}= \pm 1 / 2$ is largely split by the SO interaction, which results in a large spin polarization $P_{z}$.

\section{B. Case of $k_{\mathbf{F}} R_{0}=3$}

Figure 4 shows the calculated results for the three-terminal device in the presence of three conduction channels in the leads $\left(k_{\mathrm{F}} R_{0}=3\right)$ : (A) conductance $G_{ \pm}$for $s_{z}= \pm 1 / 2$ and (B) spin polarization $P_{z}$ in the $z$ direction, as functions of the potential depth $\left|V_{0}\right|$. Figure 4(C) shows a grayscale plot of the density of states $D(E)$ in the plane of $\left|V_{0}\right|$ and energy $E$ of electron. In Fig. 4(B), a broken line indicates the spin polarization $P_{z}$ in the four-terminal device.

The conductance $G_{ \pm}$shows several minima as a function of potential depth $\left|V_{0}\right|$. The spin polarization $P_{z}$ is enhanced around the minima of $G_{ \pm}$. These properties can be understood 

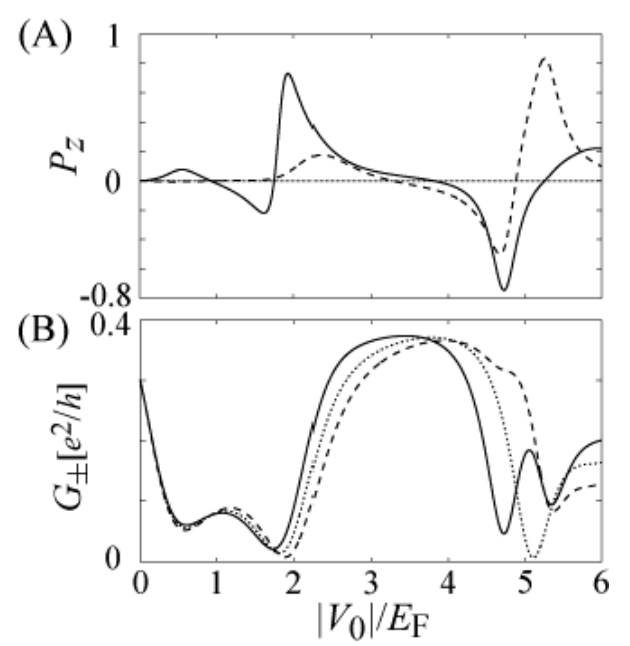

FIG. 5: A channel analysis for incident waves from reservoir 1 in the three-terminal device with $k_{\mathrm{F}} R_{0}=2$. (A) Spin polarization $P_{z}$ of the output current in reservoir 2 in Fig. 1(A), as a function of the potential depth $\left|V_{0}\right|$, for the incident electrons in the lowest channel (solid line) and second channel (broken line). (B) Conductance $G_{ \pm}$from reservoir 1 to 2 for the incident electrons in the lowest channel with $s_{z}=1 / 2$ (solid line) and $-1 / 2$ (broken line). A dotted line indicates the conductance per spin in the absence of the SO interaction.

in the same way as in the preceding subsection. The polarization $P_{z}$ is enhanced to $41 \%$ at $\left|V_{0}\right| / E_{\mathrm{F}}=3.1$ in the threeterminal device, and it is enhanced to $49 \%$ at $\left|V_{0}\right| / E_{\mathrm{F}}=3.2$ in the four-terminal device. This is due to resonant scattering via $G$-like virtual bound states $\left(l_{z}= \pm 4\right)$.

Compared with the case of two conduction channels in Figs. 2] and 3, the values of the conductance $G_{ \pm}$are larger in the case of three conduction channels $\left(k_{\mathrm{F}} R_{0}=3\right)$, whereas the maximum value of spin polarization is almost the same. This implies a more efficient spin filter in the case of three conduction channels than in the case of two conduction channels.

\section{Channel analysis for spin filtering}

In cases of $k_{\mathrm{F}} R_{0}=2$ and 3 , there are two and three conduction channels in the leads, respectively. To examine the resonant scattering in detail, we perform a channel analysis of incident waves from reservoir 1 . The results are given only for the three-terminal device in this subsection.

In the case of $k_{\mathrm{F}} R_{0}=2$, we plot the spin polarization $P_{z}$ for the incident electrons in the lowest and second channels in Fig. 5(A). At $\left|V_{0}\right| / E_{\mathrm{F}} \sim 2$ (resonance by $D$-like virtual bound state), $P_{z}$ is enhanced to $73 \%$ for the lowest channel while it is to $18 \%$ for the second channel. Hence the former plays a main role in the spin polarization. At $\left|V_{0}\right| / E_{\mathrm{F}} \sim 5$ (resonance by $F$-like virtual bound state), on the other hand, $\left|P_{z}\right|$ becomes $75 \%$ for the lowest channel while it becomes $83 \%$ for the second channel. Then both channels are important for the spin-dependent scattering.

We could selectively inject the lowest channel to the junc-
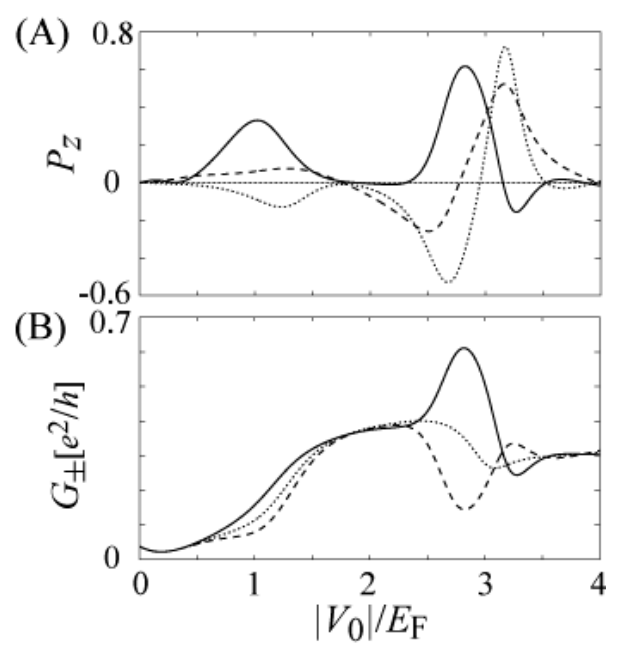

FIG. 6: A channel analysis for incident waves from reservoir 1 in the three-terminal device with $k_{\mathrm{F}} R_{0}=3$. (A) Spin polarization $P_{z}$ of the output current in reservoir 2 in Fig. 11A), as a function of the potential depth $\left|V_{0}\right|$, for the incident electrons in the lowest channel (solid line), second channel (broken line), and third channel (dotted line). (B) Conductance $G_{ \pm}$from reservoir 1 to 2 for the incident electrons in the lowest channel with $s_{z}=1 / 2$ (solid line) and $-1 / 2$ (broken line). A dotted line indicates the conductance per spin in the absence of the SO interaction.

tion, e.g., using a quantum point contact fabricated on the lead connected to reservoir 1 . Then we could realize a spin filter with an efficiency of about 75\%. In Fig.5.B), we plot the conductance $G_{ \pm}$when only the lowest channel is injected from reservoir 1. At $\left|V_{0}\right| / E_{\mathrm{F}} \sim 2$, the conductance almost vanishes although $P_{z}$ is enhanced to $73 \%$. This is due to an interference effect at the junction as in the case of single conduction channel with $k_{\mathrm{F}} R_{0}=1$ (Appendix B). At $\left|V_{0}\right| / E_{\mathrm{F}} \sim 5$, on the other hand, the total conductance is $G_{+}+G_{-}=0.4\left(e^{2} / h\right)$ and $P_{z}=-75 \%$. The latter situation is favorable to application to a spin filter.

A similar channel analysis is given for the case of $k_{\mathrm{F}} R_{0}=3$ in Fig. 6. There are three incident channels in this case. It is notable that, at $\left|V_{0}\right| / E_{\mathrm{F}} \sim 2.8$, a spin polarization of $P_{z}=$ $62 \%$ is realized for the incident electrons in the lowest channel while the total conductance is as large as $G_{+}+G_{-}=0.8\left(e^{2} / h\right)$.

\section{Repulsive potential}

We investigate the SHE caused by the scattering by a repulsive potential, $V_{0}>0$ in Eq. (6). Figure7 7 shows (A) conductance $G_{ \pm}$for $s_{z}= \pm 1 / 2$ and (B) spin polarization $P_{z}$ in the $z$ direction, when the potential height $V_{0}$ is gradually increased. The extrinsic SHE is expected even with a repulsive potential $\stackrel{18}{.}$ However, the spin-filtering effect is much weaker than the case with an attractive potential. In Fig. 7(B), the spin polarization is at most $P_{z} \approx 0.3 \%$ in the three-terminal device and $P_{z} \approx 0.45 \%$ in the four-terminal device. In this case, the resonant scattering does not take place since virtual bound 


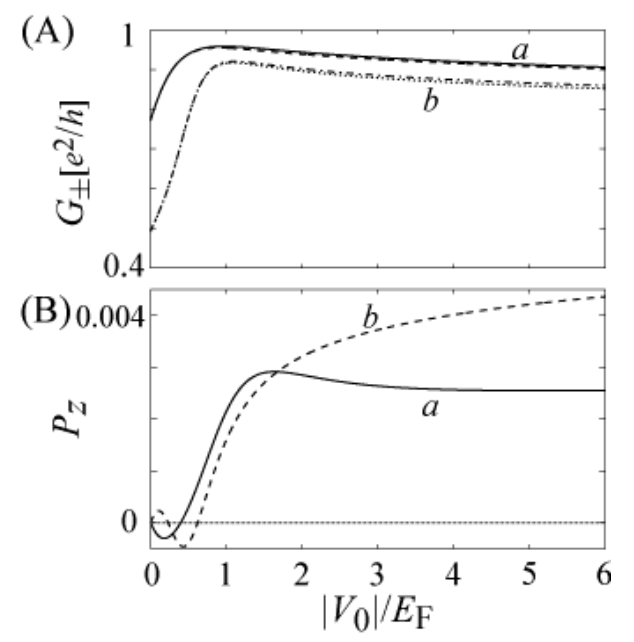

FIG. 7: Numerical results for the three-terminal device (curves labeled by $a$ ) and four-terminal device (curves labeled by $b$ ) with repulsive potential [ $V_{0}>0$ in Eq. [6]. $k_{\mathrm{F}} R_{0}=2$, where $R_{0}$ is the radius of the potential. (A) Conductance $G_{ \pm}$from reservoir 1 to 2 in Fig. 11 as a function of potential height $V_{0}$, for $s_{z}=1 / 2$ (solid lines) and $-1 / 2$ (broken lines). (B) Spin polarization $P_{z}$ of the output current in reservoir 2 .

states are hardly formed in the potential barrier. This indicates an important role of resonant scattering in the enhancement of the SHE discussed in the preceding subsections.

\section{CONCLUSIONS AND DISCUSSION}

We have numerically studied the extrinsic SHE in multiterminal devices including an antidot, fabricated on semiconductor heterostructures with strong SO interaction. The antidot creates a tunable potential on two-dimensional electron gas in the heterostructures, which may be attractive as well as repulsive. When an attractive potential is tuned properly, the resonant scattering via a virtual bound state takes place, which makes minima of the conductance from reservoir 1 to 2 in Fig. 1 Then the difference between the conductances for $s_{z}= \pm 1 / 2$ is enlarged and, as a result, the spin polarization is significantly enhanced in the direction perpendicular to the two-dimensional plane. The spin polarization can be more than $50 \%$ in our three- and four-terminal devices.

The enhancement of the extrinsic SHE by resonant scattering has been studied in different systems. Kiselev and Kim have proposed a three-terminal spin filter without antidot in the presence of Rashba SO interaction [Eq. (2)].$^{10,11}$ They have pointed out that the spin-filtering effect is enhanced by resonant scattering at the junction area when the Fermi energy of $2 \mathrm{DEG}$ is tuned. In their device, the direction of spin polarization is tilted from the $z$ direction perpendicular to the plane. In our device, the spin is polarized in the $z$ direction, which is easier to detect by an optical experiment on the Kerr rotation ${ }^{28}$ and, above all, more suitable to spintronic devices.

The extrinsic SHE enhanced by (many-body) resonant scat- tering has been examined for metallic systems with magnetic impurities ${ }^{50,51,52}$ In the case of semiconductor heterostructures, however, we have a great advantage in the tunability of scattering potential. The enhanced SHE caused by resonant scattering at a single potential can be investigated in details.

We make some comments regarding our calculations. (i) The electron-electron interaction has been neglected. Below the band edge of the lowest conduction channel in the leads, we have observed bound states in the density of states in the potential well. Since the bound states are occupied by electrons, we have to consider the electron-electron interaction between the electrons and conduction electrons at the Fermi level. The Hartree potential from the trapped electrons should be taken into account although the Coulomb blockade is irrelevant to the case of antidot potential without tunnel barriers, in contrast to the case of conventional quantum dots $\stackrel{35}{3}$ In our calculated results, therefore, the values of $\left|V_{0}\right|$ at the resonance are underestimated.

(ii) It is necessary to create such a deep potential as $\left|V_{0}\right| \sim$ $E_{\mathrm{F}}$ in designing the devices. This might be difficult with a usual antidot structure fabricated on semiconductor heterostructures. Alternatively, we could make such a deep potential using a STM tip, a charged impurity under the antidot, etc.

(iii) We have assumed that the antidot potential $V(\boldsymbol{r})$ is independent of $z$. Otherwise, the Rashba-type SO interaction, Eq. (2) with $\alpha=\lambda(\partial V / \partial z)$, must be added to Eq. (4). This would create an effective magnetic field in the $x y$ plane and thus decrease the spin polarization in the $z$ direction. The Dresselhaus SO interaction has also been disregarded. The SO interaction is induced by the inversion asymmetry of the crystal ${ }^{34}$ and expressed as

$$
H_{\mathrm{SO}}=\frac{\beta}{\hbar}\left(-p_{x} \sigma_{x}+p_{y} \sigma_{y}\right) .
$$

This would also result in an effective magnetic field in the $x y$ plane and lessen the spin polarization in the $z$ direction.

\section{ACKNOWLEDGMENTS}

This work was partly supported by the Strategic Information and Communications R\&D Promotion Program (SCOPE) from the Ministry of Internal Affairs and Communications of Japan, and by a Grant-in-Aid for Scientific Research from the Japan Society for the Promotion of Science.

\section{APPENDIX A: FORMULATION OF SPIN HALL EFFECT IN 2DEG}

Here, we summarize our previous study in Ref. 29.

First, we give a quantum mechanical formulation of the extrinsic SHE for 2DEG. For the scattering problem with Eq. (5), we adopt the partial wave expansion with $l_{z}=m=0$, $\pm 1, \pm 2, \cdots .53$ As an incident wave, we consider a plane wave propagating in the $x$ direction, $e^{i k x}$, with spin $s_{z}=1 / 2$ or 
$-1 / 2 . E=\hbar^{2} k^{2} /\left(2 m^{*}\right)$. The plane wave is expanded as

$$
e^{i k x}=e^{i k r \cos \theta}=\sum_{m=-\infty}^{\infty} i^{m} J_{m}(k r) e^{i m \theta}
$$

where $\theta$ is the angle from the $x$ direction and $J_{m}$ is the $m$ th Bessel function. Its asymptotic form at $r \rightarrow \infty$ is given by $J_{m}(k r) \sim \sqrt{2 /(\pi k r)} \cos (k r-m \pi / 2-\pi / 4)$. In the solution of Eq. (5), $J_{m}(k r)$ in Eq. (A1) is replaced by $R_{m}^{ \pm}(r)$ for $s_{z}= \pm 1 / 2$,

$$
\psi^{ \pm}(\boldsymbol{r})=\sum_{m=-\infty}^{\infty} i^{m} R_{m}^{ \pm}(r) e^{i m \theta}
$$

for spin $s_{z}= \pm 1 / 2$, where $R_{m}^{ \pm}(r)$ satisfies

$$
\begin{gathered}
{\left[-\frac{\hbar^{2}}{2 m^{*}}\left(\frac{d^{2}}{d r^{2}}+\frac{1}{r} \frac{d}{d r}-\frac{m^{2}}{r^{2}}\right)+V(r) \pm \frac{m}{2} V_{1}(r)\right] R_{m}^{ \pm}(r)} \\
=E R_{m}^{ \pm}(r) .
\end{gathered}
$$

Its asymptotic form determines the phase shift $\delta_{m}^{ \pm}$:

$$
R_{m}^{ \pm}(r) \sim \sqrt{\frac{2}{\pi k r}} e^{i \delta_{m}^{ \pm}} \cos \left(k r-\frac{m \pi}{2}-\frac{\pi}{4}+\delta_{m}^{ \pm}\right) .
$$

From Eqs. (A3) and (A4), we immediately obtain the relation of $\delta_{-m}^{ \pm}=\delta_{m}^{\mp}$, indicating the time reversal symmetry. The SO interaction is not relevant to the $S$ wave $(m=0): \delta_{0}^{+}=\delta_{0}^{-} \equiv$ $\delta_{0}$.

The scattering amplitude $f^{ \pm}(\theta)$ for spin $s_{z}= \pm 1 / 2$ is expressed in terms of phase shifts. The asymptotic form of the wavefunction in Eq. (A2) is given by

$$
\psi^{ \pm} \sim e^{i k x}+f^{ \pm}(\theta) \frac{e^{i(k r+\pi / 4)}}{\sqrt{r}},
$$

where $f^{ \pm}(\theta)$ is related to the $S$ matrix by

$$
\begin{aligned}
f^{ \pm}(\theta) & =\sum_{m} f_{m}^{ \pm} e^{i m \theta}, \\
S_{m}^{ \pm} & =1+i \sqrt{2 \pi k} f_{m}^{ \pm}=e^{2 i \delta_{m}^{ \pm}} .
\end{aligned}
$$

From these equations, we obtain

$$
f^{ \pm}(\theta)=f_{1}(\theta) \pm f_{2}(\theta),
$$

where

$$
\begin{aligned}
f_{1}(\theta)= & \frac{1}{i \sqrt{2 \pi k}}\left[e^{2 i \delta_{0}}-1\right. \\
& \left.+\sum_{m=1}^{\infty}\left(e^{2 i \delta_{m}^{+}}+e^{2 i \delta_{m}^{-}}-2\right) \cos m \theta\right], \\
f_{2}(\theta)= & \frac{1}{\sqrt{2 \pi k}} \sum_{m=1}^{\infty}\left(e^{2 i \delta_{m}^{+}}-e^{2 i \delta_{m}^{-}}\right) \sin m \theta .
\end{aligned}
$$

The scattering cross section is given by $\sigma^{ \pm}(\theta)=\left|f^{ \pm}(\theta)\right|^{2}$. Hence the spin polarization of the scattered wave in the $\theta$ direction is expressed as

$$
P_{z}=\frac{\left|f^{+}\right|^{2}-\left|f^{-}\right|^{2}}{\left|f^{+}\right|^{2}+\left|f^{-}\right|^{2}}=\frac{2 \operatorname{Re}\left(f_{1} f_{2}^{*}\right)}{\left|f_{1}\right|^{2}+\left|f_{2}\right|^{2}}
$$
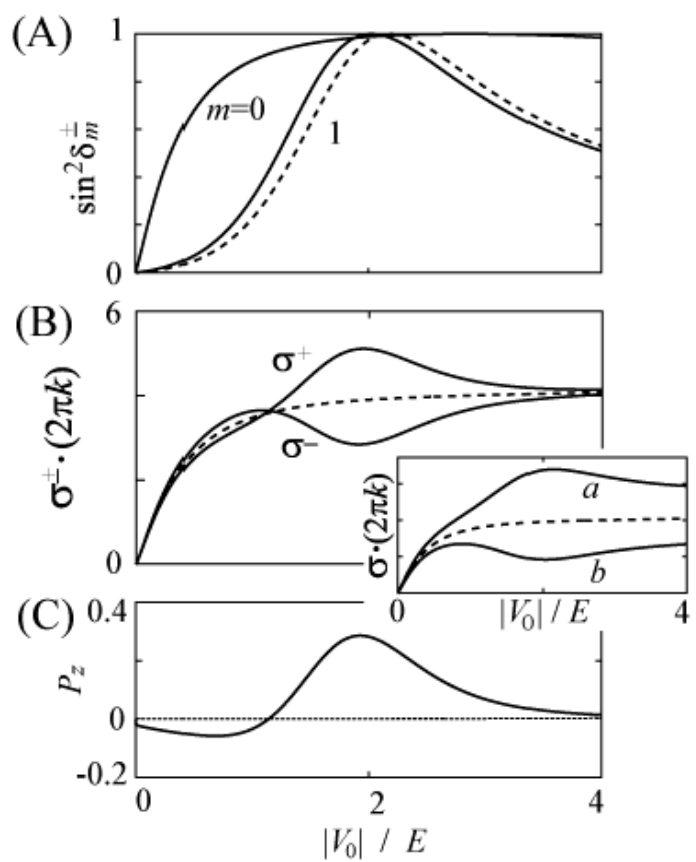

FIG. 8: Partial wave expansion for the extrinsic SHE due to the scattering by a potential well, Eq. A13, in 2DEG. $k R_{0}=1$. The strength of the SO interaction is $\lambda k^{2}=0.01$. (A) Scattering probability of each partial wave, $\sin ^{2} \delta_{m}^{ \pm}$, (B) scattering cross section $\sigma^{ \pm}(\theta=-\pi / 2)$ for $s_{z}= \pm 1 / 2$, and (C) spin polarization $P_{z}$ at $\theta=-\pi / 2$, as functions of the potential depth $\left|V_{0}\right|$ [normalized by electron energy $\left.E=\hbar^{2} k^{2} /\left(2 m^{*}\right)\right]$. In (A), solid and broken lines indicate the cases of $s_{z}=1 / 2$ and $-1 / 2$, respectively, for $m=1\left(\delta_{-1}^{ \pm}=\delta_{1}^{\mp}\right)$. The scattering probability for $|m| \geq 2$ is negligible. In (B), a broken line indicates the cross section at $\theta=-\pi / 2$ in the absence of SO interaction. Inset: scattering cross section in the absence of SO interaction at $\theta=-0.45 \pi(a),-\pi / 2$ (broken line), and $\theta=-0.55 \pi(b)$.

when the incident electron is unpolarized. This formula is analogous to that of skew scattering in three dimensions, ${ }^{26,27}$ The spin is polarized in the $z$ direction and $P_{z}(-\theta)=-P_{z}(\theta)$.

A remark is made on Eq. A5. We put a phase of $\pi / 4$ on the exponent of the second term on the right side. Although the phase has no physical meaning, it ensures the "optical theorem" that the total cross section is determined only by the amplitude of forward scattering;

$$
\sigma_{\text {total }}^{ \pm} \equiv \int_{0}^{2 \pi} \sigma^{ \pm}(\theta) d \theta=\sqrt{\frac{8 \pi}{k}} \operatorname{Im} f^{ \pm}(0) .
$$

Now we apply our formula of the SHE to the case of a potential well, Eq. (6) with $\Delta R_{0} \rightarrow 0$, that is,

$$
V(r)=V_{0} \theta\left(R_{0}-r\right)
$$

$\left(V_{0}<0\right)$, where $\theta(t)$ is a step function $[\theta(t)=1$ for $t>0,0$ for $t<0]$. Then $V_{1}=-(2 \lambda / r) V^{\prime}(r)=\left(2 \lambda / R_{0}\right) V_{0} \delta\left(r-R_{0}\right)$ with the $\delta$ function $\delta(t)$.

The phase shifts $\delta_{m}^{ \pm}$are calculated by solving Eq. A3]. The calculation is elementary. We have only to consider the case 

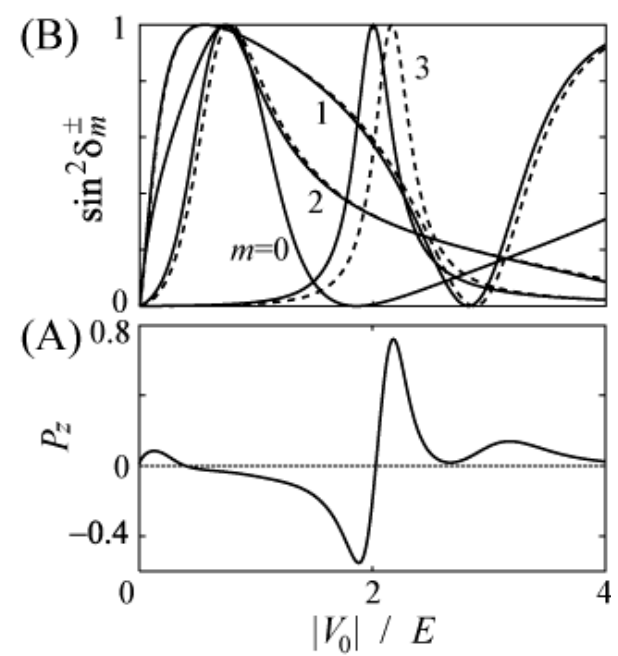

FIG. 9: Partial wave expansion for the extrinsic SHE due to the scattering by a potential well, Eq. A13, in 2DEG. $k R_{0}=2$. The strength of the SO interaction is $\lambda k^{2}=0.01$. (A) sScattering probability of each partial wave, $\sin ^{2} \delta_{m}^{ \pm}$, and (B) spin polarization $P_{z}$ at $\theta=-\pi / 2$, as functions of the potential depth $\left|V_{0}\right|$ [normalized by electron energy $\left.E=\hbar^{2} k^{2} /\left(2 m^{*}\right)\right]$. In (A), solid and broken lines indicate the cases of $s_{z}=1 / 2$ and $-1 / 2$, respectively, for $m>0\left(\delta_{-m}^{ \pm}=\delta_{m}^{\mp}\right)$.

with $m \geq 0$ because of the relation of $\delta_{-m}^{ \pm}=\delta_{m}^{\mp}$. For $r>R_{0}$, $V=0$ and thus

$$
\begin{aligned}
R_{m}^{ \pm}(r)= & C_{1} J_{m}(k r)+C_{2} Y_{m}(k r) \\
\sim & \sqrt{\frac{2}{\pi k r}}\left[C_{1} \cos (k r-m \pi / 2-\pi / 4)\right. \\
& \left.+C_{2} \sin (k r-m \pi / 2-\pi / 4)\right],
\end{aligned}
$$

where $Y_{m}$ is the $m$ th Neumann function. From Eqs. A15) and (A4), we find

$$
\tan \delta_{m}^{ \pm}=-C_{2} / C_{1} .
$$

For $r<R_{0}, V=V_{0}$. Then

$$
\begin{aligned}
R_{m}^{ \pm}(r) & =C_{3} J_{m}\left(k^{\prime} r\right), \\
\hbar^{2} k^{\prime 2} /\left(2 m^{*}\right) & =E-V_{0} .
\end{aligned}
$$

The connection of Eqs. (A14) and A17) at $r=R_{0}$ yields

$$
\tan \delta_{m}^{ \pm}=\frac{\left[J_{m-1}\left(k R_{0}\right)-J_{m+1}\left(k R_{0}\right)\right] J_{m}\left(k^{\prime} R_{0}\right)-\alpha_{m}^{ \pm} J_{m}\left(k R_{0}\right)}{\left[Y_{m-1}\left(k R_{0}\right)-Y_{m+1}\left(k R_{0}\right)\right] J_{m}\left(k^{\prime} R_{0}\right)-\alpha_{m}^{ \pm} Y_{m}\left(k R_{0}\right)}
$$

with

$$
\begin{aligned}
\alpha_{m}^{ \pm} & =\left(k^{\prime} / k\right)\left[J_{m-1}\left(k^{\prime} R_{0}\right)-J_{m+1}\left(k^{\prime} R_{0}\right)\right] \\
& \mp 2 m\left[1+\left(k^{\prime} / k\right)^{2}\right]\left(k \lambda / R_{0}\right) J_{m}\left(k^{\prime} R_{0}\right) .
\end{aligned}
$$

Here, we have used the relation of $d J_{m}(x) / d x=\left[J_{m-1}(x)-\right.$ $\left.J_{m+1}(x)\right] / 2$ and $d Y_{m}(x) / d x=\left[Y_{m-1}(x)-Y_{m+1}(x)\right] / 2$.

The calculated results are shown in Fig. 8. (A) scattering probability of each partial wave, $\sin ^{2} \delta_{m}^{ \pm}$, (B) scattering cross section $\sigma^{ \pm}(\theta=-\pi / 2)$ for $s_{z}= \pm 1 / 2$, and (C) spin polarization $P_{z}$ at $\theta=-\pi / 2$, when the potential depth $\left|V_{0}\right|$ is gradually changed. The strength of the SO interaction is set to be $\lambda k^{2}=0.01$, which corresponds to the value for InAs,

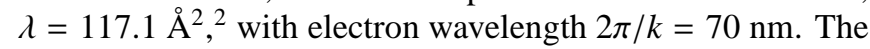
radius of the potential well is $R_{0}=1 / k$. As seen in Fig. 8 (A), with an increase in $\left|V_{0}\right|$, the scattering probability increases and becomes unity at some values of $\left|V_{0}\right|$ (unitary limit with $\left.\delta_{m}^{ \pm}=\pi / 2\right)$ for $m=0(S$ wave $)$ and $m= \pm 1$ ( $P$ wave $)$. This is due to resonant scattering through virtual bound states in the potential well. The resonant width is narrower for larger $|m|$ because of the centrifugal potential $\propto \mathrm{m}^{2} / r^{2}$ separating the bound states from the outer region.

As discussed in the text with Eq. (5), the scattering of a partial wave with positive $m$ is enhanced (suppressed) for spin $s_{z}=1 / 2\left(s_{z}=-1 / 2\right)$. Around the resonance of the $P$ waves, the scattering of $\left(m, s_{z}\right)=(1,1 / 2)$ goes to the unitary limit at a smaller value of $\left|V_{0}\right|$ than the scattering of $\left(m, s_{z}\right)=(1,-1 / 2)$. Thus we observe a difference between $\delta_{1}^{+}$and $\delta_{1}^{-}$in Fig. 8 (A). This leads to the difference of the scattering cross section at $\theta=-\pi / 2$ for $s_{z}= \pm 1 / 2$ [Fig. [8(B)] and a spin polarization $\left[P_{z} \approx 30 \%\right.$ in Fig. $\left.8(\mathrm{C})\right]$. When $\delta_{m}^{ \pm}(|m| \geq 2)$ is negligible, Eqs. (A8)-(A10) yield

$$
\begin{aligned}
\sigma^{ \pm}(\theta= & -\pi / 2)=\frac{2}{\pi k}\left[\sin ^{2} \delta_{0}+\sin ^{2} \Delta \delta_{1}\right. \\
& \left. \pm 2 \sin \delta_{0} \sin \left(2 \bar{\delta}_{1}-\delta_{0}\right) \sin \Delta \delta_{1}\right],
\end{aligned}
$$

where $\Delta \delta_{1}=\delta_{1}^{+}-\delta_{1}^{-}$and $\bar{\delta}_{1}=\left(\delta_{1}^{+}+\delta_{1}^{-}\right) / 2$. Around the resonance, $\Delta \delta_{1}$ is enlarged, which results in the enhancement of the SHE. For $\bar{\delta}_{1} \approx \pi / 2$, the spin polarization is given by

$$
P_{z}(\theta=-\pi / 2) \approx \frac{2 \sin ^{2} \delta_{0} \sin \Delta \delta_{1}}{\sin ^{2} \delta_{0}+\sin ^{2} \Delta \delta_{1}}
$$

from Eq. A11).

In our three- and four-terminal devices, the resonant scattering makes minima of the conductance. It should be hard to say, however, whether the resonant scattering enhances or suppresses the conductance in general. For simplicity, let us ignore the $\mathrm{SO}$ interaction. We plot the scattering cross section in the inset in Fig. 8 8 (B), in the direction of $\theta=-0.45 \pi,-\pi / 2$, and $\theta=-0.55 \pi$. Using $\delta_{0}$ and $\delta_{1}^{+}=\delta_{1}^{-} \equiv \delta_{1}$, the cross section is written as

$$
\begin{gathered}
\sigma(\theta)=\frac{2}{\pi k}\left[\sin ^{2} \delta_{0}+4 \sin ^{2} \delta_{1} \cos ^{2} \theta\right. \\
\left.+4 \sin \delta_{0} \sin \delta_{1} \cos \left(\delta_{0}-\delta_{1}\right) \cos \theta\right]
\end{gathered}
$$

The resonance of the $P$ wave $\left(\delta_{1}=\pi / 2\right)$ has no effect on the scattering at $\theta=-\pi / 2$. Its effect on the scattering at $\theta \neq$ $-\pi / 2$ depends on the value of $\delta_{0}$. In the inset in Fig. 8 (B), the resonance of the $P$ wave makes a peak of $\sigma(\theta=-0.45 \pi)$ and a dip of $\sigma(\theta=-0.55 \pi)$. In the situation of our devices, the conductance should reflect an integrated value of $\sigma(\theta)$ around $\theta=-\pi / 2$, and also a complicated interference effect.

Figure 9 shows the calculated results in the case of $k R_{0}=2$. The resonant scattering is observed for $0 \leq|m| \leq 3$. Around the resonance of $F$ waves $(|m|=3), P_{z}$ is enhanced to $72 \%$. 


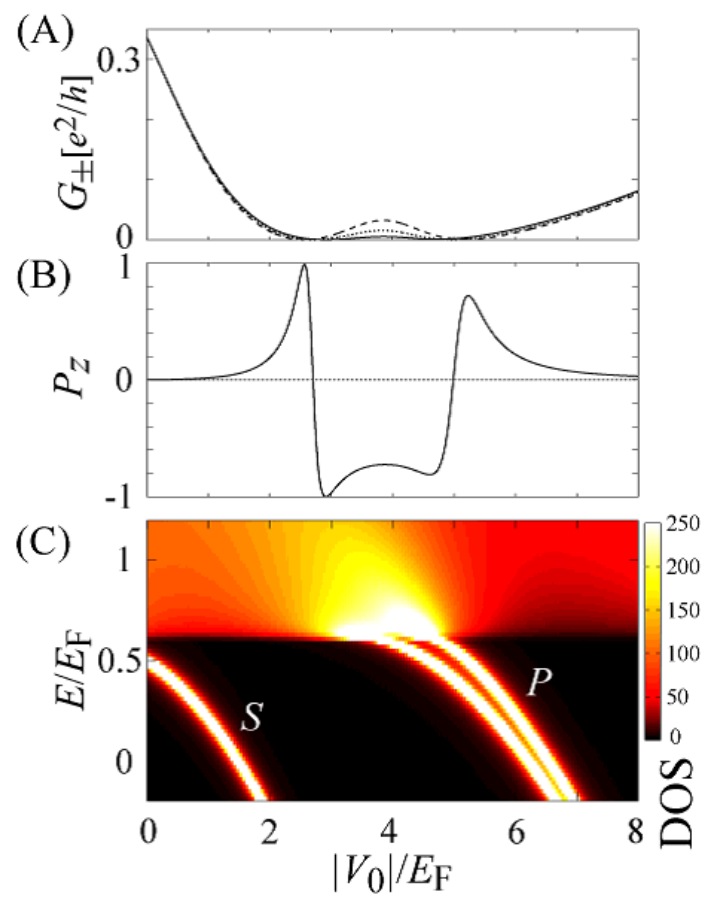

FIG. 10: (Color online) Numerical results for the three-terminal device with $k_{\mathrm{F}} R_{0}=1$, where $R_{0}$ is the radius of attractive potential. (A) Conductance $G_{ \pm}$from reservoir 1 to 2 in Fig. 11 A) for $s_{z}= \pm 1 / 2$ and (B) spin polarization $P_{z}$ of the output current in reservoir 2, as functions of the potential depth $\left|V_{0}\right|$. In (A), solid and broken lines indicate $G_{+}$and $G_{-}$, respectively. A dotted line shows the conductance per spin in the absence of the SO interaction. (C) Grayscale plot of the density of states in the junction area, $D(E)$, in the plane of $\left|V_{0}\right|$ and energy $E$ of electron.

In general, a sharper resonance enlarges $\delta_{m}^{+}-\delta_{m}^{-}$for larger $|m|$, which results in a larger polarization.

In Fig. 9(B), $P_{z}$ shows a dip and peak structure at the $F$ wave resonance. This structure is observed when the resonance of $\left(m, s_{z}\right)=(3,1 / 2)$ is sufficiently separated from that of $\left(m, s_{z}\right)=(3,-1 / 2)$. Around the $F$-wave resonance, $\delta_{1}^{+} \approx \delta_{1}^{-}$ and $\delta_{2}^{+} \approx \delta_{2}^{-}\left(\equiv \delta_{2}\right)$, as seen in Fig. 9 (A). Neglecting $\delta_{0}$, Eq. (A11) yields

$$
P_{z}(\theta=-\pi / 2) \approx \frac{4 \sin \delta_{2} \sin \left(2 \bar{\delta}_{3}-\delta_{2}\right) \sin \Delta \delta_{3}}{4 \sin ^{2} \delta_{2}+\sin ^{2} \Delta \delta_{3}},
$$

where $\Delta \delta_{3}=\delta_{3}^{+}-\delta_{3}^{-}$and $\bar{\delta}_{3}=\left(\delta_{3}^{+}+\delta_{3}^{-}\right) / 2$. At the resonance of $\left(m, s_{z}\right)=(3,1 / 2), \delta_{3}^{+}=\pi / 2$ and $\delta_{3}^{-} \approx 0$. Then $P_{z}(\theta=-\pi / 2)$ shows a dip for $\pi / 2<\delta_{2}<\pi$. At the resonance of $\left(m, s_{z}\right)=$ $(3,-1 / 2), \delta_{3}^{+} \approx \pi$ and $\delta_{3}^{-}=\pi / 2$. Then $P_{z}(\theta=-\pi / 2)$ shows a peak. A similar dip-peak structure of $P_{z}$ is observed around $F$-wave resonance in Figs. 2 and 3 for our devices.

\section{APPENDIX B: CASE OF $k_{\mathrm{F}} R_{0}=1$}

We present calculated results in the case of attractive potential and $k_{\mathrm{F}} R_{0}=1$, where the number of conduction channels is unity in the leads. In this case, an interference effect around the junction strongly influences the conductance and spin polarization. We discuss the results only for the three-terminal device.

Figures 10.(A) and 10(B) show the conductance $G_{ \pm}$for $s_{z}= \pm 1 / 2$ and spin polarization $P_{z}$, respectively, when the potential depth $\left|V_{0}\right|$ is gradually changed. As seen in Fig.10(A), the conductance $G_{ \pm}$vanishes two times at $\left|V_{0}\right| / E_{\mathrm{F}} \approx 2.7$ and 5.0. The reason why the conductance completely disappears cannot be explained by resonant scattering only. This is due to an interference effect around the junction area. The value of $\left|V_{0}\right|$ for $G_{+}=0$ and that for $G_{-}=0$ are different from each other, in the presence of the $\mathrm{SO}$ interaction. As a result, $P_{z}=1$ at $G_{-}=0$ and $P_{z}=-1$ at $G_{+}=0$, as seen in Fig. 10(B).

Figure 10(C) is a grayscale plot of the density of states in the junction area, $D(E)$, in the plane of $\left|V_{0}\right|$ and energy $E$ of electron. The band edge of the lowest conduction channel in the leads is at $E / E_{\mathrm{F}}=0.616$. A virtual bound state of $P$ like state is seen at the Fermi level at the first zero point of the conductance $\left(\left|V_{0}\right| / E_{\mathrm{F}} \approx 2.7\right)$. This indicates a resonant scattering via the virtual bound state there. However, we do not observe any virtual bound state at the second zero point.

We observe a perfect spin polarization of $P_{z}= \pm 1$ in Fig. 10(B). However, the absolute value of the conductance is very small when $P_{z}= \pm 1$. Hence, it should be difficult to apply this situation to a spin filter.
* E-mail me at: tyokoyam@kobrmc34.rk.phys.keio.ac.jp

${ }^{1}$ I. Žutić, J. Fabian, and S. Das Sarma, Rev. Mod. Phys. 76, 323 (2004).

2 R. Winkler, Spin-Orbit Coupling Effects in Two-Dimensional Electron and Hole Systems (Springer, Berlin Heidelberg, 2003).

${ }^{3}$ E. I. Rashba, Fiz. Tverd. Tela (Leningrad) 2, 1224 (1960); Yu. A. Bychkov and E. I. Rashba, J. Phys. C 17, 6039 (1984).

4 J. Nitta, T. Akazaki, H. Takayanagi, and T. Enoki, Phys. Rev. Lett. 78, 1335 (1997).

5 D. Grundler, Phys. Rev. Lett. 84, 6074 (2000).
6 Y. Sato, T. Kita, S. Gozu, and S. Yamada, J. Appl. Phys. 89, 8017 (2001).

7 S. Datta and B. Das, Appl. Phys. Lett. 56, 665 (1990).

${ }^{8}$ G. Schmidt, D. Ferrand, L. W. Molenkamp, A. T. Filip, and B. J. van Wees, Phys. Rev. B 62, R4790 (2000).

9 E. N. Bulgakov, K. N. Pichugin, A. F. Sadreev, P. Streda, and P. Seba, Phys. Rev. Lett. 83, 376 (1999).

10 A. A. Kiselev and K. W. Kim, Appl. Phys. Lett. 78, 775 (2001).

11 A. A. Kiselev and K. W. Kim, J. Appl. Phys. 94, 4001 (2003).

12 T. P. Pareek, Phys. Rev. Lett. 92, 076601 (2004). 
13 T. Koga, J. Nitta, H. Takayanagi, and S. Datta, Phys. Rev. Lett. 88, 126601 (2002).

${ }^{14}$ M. Eto, T. Hayashi, and Y. Kurotani, J. Phys. Soc. Jpn. 74, 1934 (2005).

15 P. G. Silvestrov and E. G. Mishchenko, Phys. Rev. B 74, 165301 (2006).

16 J. I. Ohe, M. Yamamoto, T. Ohtsuki, and J. Nitta, Phys. Rev. B 72, 041308(R) (2005).

17 J. J. Krich and B. I. Halperin, Phys. Rev. B 78, 035338 (2008).

18 M. Yamamoto and B. Kramer, J. Appl. Phys. 103, 123703 (2008).

19 S. Murakami, N. Nagaosa, and S.-C. Zhang, Science 301, 1348 (2003).

20 J. Sinova, D. Culcer, Q. Niu, N. A. Sinitsyn, T. Jungwirth, and A. H. MacDonald, Phys. Rev. Lett. 92, 126603 (2004).

21 J. Wunderlich, B. Kaestner, J. Sinova, and T. Jungwirth, Phys. Rev. Lett. 94, 047204 (2005).

${ }^{22}$ M. I. Dyakonov and V. I. Perel, Phys. Lett. 35A, 459 (1971).

23 J. E. Hirsch, Phys. Rev. Lett. 83, 1834 (1999).

${ }^{24}$ S. Zhang, Phys. Rev. Lett. 85, 393 (2000).

25 H. A. Engel, B. I. Halperin, and E. I. Rashba, Phys. Rev. Lett. 95, 166605 (2005).

${ }^{26}$ N. F. Mott and H. S. Massey, Theory of Atomic Collisions, 3rd ed. (Oxford, 1965).

27 L. D. Landau and E. M. Lifshitz, Quantum Mechanics (Course of theoretical physics, Vol. 3) 3rd ed. (Pergamon Press, 1977).

28 Y. K. Kato, R. C. Myers, A. C. Gossard, and D. D. Awschalom, Science 306, 1910 (2004).

${ }^{29}$ M. Eto and T. Yokoyama, J. Phys. Soc. Jpn. 78, 073710 (2009).

${ }^{30}$ K. Hattori and H. Okamoto, Phys. Rev. B 74, 155321 (2006).

31 S. Bellucci and P. Onorato, Phys. Rev. B 74, 245314 (2006).

32 Y. Jiang and L. Hu, Phys. Rev. B 74, 075302 (2006).

33 Y. Xing, Q. F. Sun, L. Tang, and J. P. Hu, Phys. Rev. B 74, 155313 (2006).

${ }^{34}$ G. Dresselhaus, Phys. Rev. 100, 580 (1955).

35 L. P. Kouwenhoven, C. M. Marcus, P. L. McEuen, S. Tarucha, R. M. Westervelt, and N. S. Wingreen, in Mesoscopic Elec- tron Transport, NATO ASI Series E 345, eds. L. Y. Sohn, L. P. Kouwenhoven, and G. Schön (Kluwer, Dordrechit, 1997), p. 105.

36 T. Aono, Phys. Rev. Lett. 93, 116601 (2004), and references cited therein for the spin pumping.

37 D. Feinberg and P. Simon, Appl. Phys. Lett. 85, 1846 (2004).

38 M. Pustilnik and L. Borda, Phys. Rev. B 73, 201301(R) (2006).

39 P. Sharma and C. Chamon, Phys. Rev. Lett. 87, 096401 (2001).

${ }^{40}$ R. Citro, N. Andrei, and Q. Niu, Phys. Rev. B 68, 165312 (2003).

${ }^{41}$ M. Pustilnik, E. G. Mishchenko, and O. A. Starykh, Phys. Rev. Lett. 97, 246803 (2006).

42 B. Braunecker, D. E. Feldman, and F. Li, Phys. Rev. B 76, 085119 (2007).

43 Related to the quantum wires, we also refer a paper on a spin filter using the spin edge states of quantum Hall effect in graphene; D. A. Abanin, P. A. Lee, and L. S. Levitov, Phys. Rev. Lett. 96, 176803 (2006).

44 S. Dtta, Electronic Transport in Mesoscopic Systems (Cambridge University Press, Cambridge, 1995).

45 T. Ando, Phys. Rev. B 40, 5325 (1989).

46 T. Ando, Phys. Rev. B 44, 8017 (1991).

47 M. Yamamoto, T. Ohtsuki, and B. Kramer, Phys. Rev. B 72, 115321 (2005).

48 For the calculation of DOS, a small imaginary part, $i E_{\mathrm{F}} / 100$, is added to $E$ in Eq. (14), to broaden the sharp peaks corresponding to the bound states.

49 This dispersion relation is obtained from Eq. [12) if the cosineband is approximated by the parabolic band (wide limit of the leads).

50 A. Fert and O. Jaoul, Phys. Rev. Lett. 28, 303 (1972).

51 A. Fert, A. Friederich, and A. Hamzic, J. Magn. Magn. Mater. 24, 231 (1981).

52 G. Y. Guo, S. Maekawa, and N. Nagaosa, Phys. Rev. Lett. 102, 036401 (2009), and related references cited therein.

53 Y. Aharonov and D. Bohm, Phys. Rev. 115, 485 (1959). 